\title{
Revista Colombiana de

\section{Tratamiento del síncope neuralmente mediado con marcapasos: utilidad del sensor de asa cerrada}

\author{
Martín de la Ossa ${ }^{a}$, Mauricio Duque ${ }^{\mathrm{a}, *}$ y Laura Duque ${ }^{\mathrm{b}}$ \\ a Servicio CES Cardiología, Clínica CES, Medellín, Colombia \\ ${ }^{\mathrm{b}}$ Estudiante Facultad de Medicina, Universidad CES, Medellín, Colombia
}

Recibido el 4 de junio de 2014; aceptado el 17 de octubre de 2014

Disponible en Internet el 11 de marzo de 2015

\section{PALABRAS CLAVE \\ Marcapasos; \\ Síncope; \\ Estimulación \\ ventricular}

\section{KEYWORDS \\ Pacemakers; \\ Syncope; \\ Ventricular pacing}

\begin{abstract}
Resumen El síncope vasovagal es una entidad frecuente, de difícil manejo, con alta tasa de recurrencia aun con manejo médico. Se ha estudiado la estimulación cardiaca en pacientes con respuesta cardioinhibitoria en la mesa basculante con resultados contradictorios. Los estudios iniciales mostraron buenos resultados, que no lograron reproducirse cuando se introdujo el diseño doble ciego. La mayoría de estos estudios se realizaron con marcapasos con sensores convencionales. Las guías actuales de dispositivos indican la terapia de estimulación cardiaca en síncope vasovagal con respuesta cardioinhibitoria como una alternativa ante la no respuesta al tratamiento convencional. Existe evidencia reciente que indica que los marcapasos con sensores de asa cerrada (CLS, del inglés closed-loop sensor) muestran mejores resultados que los sensores convencionales; estos estudios, aunque con población pequeña, reportan reducciones de la frecuencia de síncopes y presíncopes. Este tipo de dispositivos actúan en etapas más tempranas de la cascada de eventos fisiopatológicos del síncope vasovagal, detectando cambios en la impedancia ventricular antes de la caída de la frecuencia cardiaca, lo cual permite intervenir en forma precoz con estimulación para evitar el síncope.

๑) 2015 Sociedad Colombiana de Cardiología y Cirugía Cardiovascular. Publicado por Elsevier España, S.L.U. Este es un artículo Open Access bajo la licencia CC BY-NC-ND (http://creativecommons.org/licenses/by-nc-nd/4.0/).
\end{abstract}

Neurally mediated syncope treatment with pacemakers: Utility of closed-loop sensor

Abstract The vasovagal syncope is a frequent entity of difficult management with a high rate of recurrence despite medical management. Cardiac pacing has been studied in patients with cardioinhibitory response on tilt table with inconsistent results. Initial studies showed beneficial results were achieved, but they could be reproduced when the double-blind design was introduced. Most of these studies were performed with conventional pacemaker sensors. Device current guidelines indicate pacing therapy in vasovagal syncope with cardioinhibitory response

\footnotetext{
* Autor para correspondencia.

Correo electrónico: mauricioduque@une.net.co (M. Duque).
} 
as an alternative to non-response to conventional therapy. There is recent evidence that pacemakers with closed-loop sensors (CLS) show better results than conventional sensors; these studies, although limited to small groups of population, show reductions in the frequency of syncopes and presyncopes. These devices work in earlier stages of the cascade of pathophysiological events of the vasovagal syncope, detecting changes in ventricular impedance before the fall in heart rate, thus allowing early intervention as stimulation to prevent syncope.

(c) 2015 Sociedad Colombiana de Cardiología y Cirugía Cardiovascular. Published by Elsevier España, S.L.U. This is an open access article under the CC BY-NC-ND license (http://creativecommons.org/licenses/by-nc-nd/4.0/).

\section{Introducción}

El síncope es una pérdida de conciencia, con inicio y recuperación rápida y completa, secundaria a hipoperfusión cerebral transitoria ${ }^{1}$. La hipoperfusión cerebral puede deberse a disminución de la resistencia periférica, usualmente por vasodilatación o disminución del gasto cardiaco, incluyendo como causas de esta última la bradicardia o la taquicardia. Esta patología implica una carga considerable para los sistemas de salud del mundo. Su prevalencia aumenta con la edad, variando desde 6,2 por 100 personas/año en adultos jóvenes, hasta 19 por 1.000 personas/año en mayores de 80 años $^{2}$. La incidencia incrementa cuando hay enfermedad estructural cardiaca (10,6 contra 6,4 por 1.000 pacientes/año). Con relación a la mortalidad del síncope neuralmente mediado, es la misma cuando se compara con sujetos sanos de igual edad ${ }^{2}$. El síncope representa el $1 \%$ de las consultas en los servicios de urgencias, con un promedio de hospitalización del $40 \%$, resultado que corresponde a cerca de 200.000 hospitalizaciones solo en Estados Unidos, con un costo promedio de 5.400 dólares/hospitalización ${ }^{3,4}$. El promedio de visitas de los pacientes con síncope al médico es de 10 al año, en tanto que el promedio del número de especialistas que participan en el manejo es de $3,2^{5}$.

Las recurrencias son frecuentes después de un episodio inicial. Una historia de uno o 2 episodios predice una tasa de recurrencia del 15 al $20 \%$ después de uno a 2 años, respectivamente, mientras que 3 episodios predicen una recurrencia del 36 al 42\% ${ }^{6}$. Por el contrario, el género, la severidad de la presentación y la ausencia de enfermedad estructural tienen pobre valor pronóstico en términos de recurrencia. Esta última tiene un impacto notable en la calidad de vida de los pacientes; el $76 \%$ de los afectados presentan un cambio en relación con sus actividades diarias y un $64 \%$ limitan la conducción de vehículos ${ }^{7}$. Las lesiones también son frecuentes, pues ocurren en el $30 \%$ de los pacientes admitidos con este diagnóstico, y el $5 \%$ corresponden a trauma severo ${ }^{8}$. Aproximadamente el $10 \%$ de las caídas en la población geriátrica son atribuibles a un síncope ${ }^{9}$.

El síncope vasovagal es el tipo predominante, responsable del 20 al $65 \%$ de los casos. Hay una distribución bimodal, con un pico de inicio en la adolescencia y por encima de los 65 años. El curso clínico usualmente es autolimitado, particularmente en menores de 30 años y, en general, el pronóstico es bueno. La estrategia inicial de manejo es conservadora, con énfasis en los cambios en el estilo de vida y evitando las situaciones desencadenantes con el reconocimiento temprano de los síntomas y el uso de maniobras para abortar los síntomas. Aunque algunos pacientes responden muy bien a este tipo de manejo no farmacológico, existen casos en los que no es suficiente. Infortunadamente ninguna de las terapias farmacológicas ha mostrado eficacia comprobada y las maniobras de entrenamiento son difíciles de cumplir dada la adherencia que requieren. Puede incluirse tratamiento farmacológico y estimulación permanente para los casos refractarios $2,10,11$. Adicionalmente, el implante de un marcapasos estaría claramente indicado cuando durante el síncope se documente disfunción del nodo $\mathrm{AV}$ o bloqueo $\mathrm{AV}^{12}$.

Al momento de implantar un marcapasos en un paciente con síncope neuralmente mediado se debe tener en la cuenta que el síncope es una entidad de curso errático, con episodios de exacerbación y remisión sin causa definida, y de mayor frecuencia en población joven. Así mismo, se debe comprender que la bradicardia, aun con asistolia prolongada, solo es una parte del mecanismo, y en ocasiones esta no se relaciona con los síntomas del paciente, de ahí que el tratamiento aislado de la misma correspondería a un manejo incompleto. En los casos donde predomina la respuesta vasodilatadora, la estimulación cardiaca es poco efectiva en prevenir la recurrencia del síncope; no obstante, en los casos de respuesta mixta, especialmente en aquellos con asistolia prolongada, la estimulación cardiaca pudiera ofrecer algunas ventajas. Sobre este último punto existe controversia en la literatura médica, dados los resultados disímiles de los múltiples estudios sobre el tema ${ }^{13}$. El objetivo de este artículo es revisar la evidencia actual sobre el uso de marcapasos en pacientes con síncope mediado neuralmente, haciendo énfasis en el sistema de sensores de asa cerrada (CLS, del inglés closed-loop sensor).

\section{Fisiopatología del síncope vasovagal}

El síncope vasovagal es causado principalmente por deterioro agudo con interacciones complejas entre el sistema nervioso autónomo y el cardiovascular encargados de mantener la perfusión cerebral. La activación inapropiada del reflejo de Bezold-Jarisch, por la estimulación de los mecanorreceptores localizados en el ventrículo izquierdo, debería ser primariamente responsable de este fenómeno. Cada paciente con síncope vasovagal presenta un mecanismo combinado de vasodepresión y cardioinhibición que causa el evento, en el que a la respuesta cardioinhibito- 
ria (con y sin completa asistolia) usualmente le sigue un incremento en la contractilidad miocárdica ${ }^{14}$.

La característica principal del síncope es la reducción de la presión arterial sistémica, con la correspondiente interrupción del flujo sanguíneo cerebral. Está documentado que solo 6 segundos (s) de reducción del flujo sanguíneo cerebral son suficientes para generar pérdida de conciencia. A nivel práctico, en la mesa basculante la disminución de presión sistólica a menos de $60 \mathrm{mmHg}$ se asocia con síncope ${ }^{15}$.

El síncope neuralmente mediado puede clasificarse como vasodepresor cuando predomina la hipotensión debida a la pérdida del tono vasoconstrictor, cardioinhibitorio cuando predomina la bradicardia o la asistolia, y mixto cuando están presentes los 2 mecanismos ${ }^{16}$.

La reacción vasovagal es consecuencia de una reducción súbita del volumen sanguíneo. La hipovolemia central puede deberse al deterioro de la venoconstricción y a la ineficiencia del tono esplácnico, así como de otros vasos de resistencia. Lo anterior induce un incremento compensatorio en la descarga simpática, con aumento en la frecuencia cardiaca e inotropismo miocárdico. La contracción más vigorosa del miocardio en el contexto de un llenado ineficiente estimula los mecanorreceptores ventriculares. Las señales aferentes de estos receptores se transmiten a través del nervio vago, alcanzan los centros circulatorios y disparan un aumento en la actividad vagal eferente y una suspensión de la descarga simpática eferente. Estas señales desencadenadas originadas en el miocardio conducen a hipotensión y bradicardia (reflejo de Benzold-Jarisch), lo cual es reproducido en la mesa basculante ${ }^{17}$.

La respuesta vasodepresora se caracteriza por una caída de la presión sistólica, con una caída de la frecuencia cardiaca al momento del síncope menor al $10 \%$ de su valor pico. En la respuesta mixta, la presión sanguínea cae antes de la frecuencia cardiaca; la frecuencia ventricular no cae a menos de 40 latidos por minuto (lpm) o cae a menos de ese valor por menos de $10 \mathrm{~s}$ con o sin asistolia (menor de $3 \mathrm{~s}$ ). Si la bradicardia y/o la asistolia son el mecanismo predominante, la respuesta de la mesa basculante se clasifica como cardioinhibitoria.

En presencia de una respuesta vasopresora pura, la estimulación cardiaca no tiene cabida. Su papel sería significativo en los casos de respuesta cardioinhibitoria, especialmente ante asistolia prolongada.

\section{Manejo con dispositivos}

El síncope vasovagal frecuentemente es autolimitado. Esto tiene un impacto considerable en la decisión de indicar terapias invasivas, particularmente en la población joven. La estimulación cardiaca permanente es una forma potencial pero aun controversial de tratamiento en estos pacientes. $\mathrm{El}$ objetivo de la estimulación cardiaca se encamina al manejo de la bradicardia transitoria, de modo que se provee una frecuencia cardiaca para soportar la caída de la presión arterial que frecuentemente acompaña al síncope vasovagal. Los principales campos de atención han sido la detección oportuna del inicio del síncope y la respuesta durante el episodio. El manejo más simple sería la estimulación cardiaca con un dispositivo de una cámara para el manejo de la bradicardia que acompaña al síncope.

En términos generales, el síncope es una entidad compleja en ocasiones mal entendida, en otras de difícil interpretación y abordaje, que siempre se presenta como un reto diagnóstico y terapéutico, esto último marcado por la cronicidad de la entidad y la ausencia de un manejo definitivo a la fecha. Es sobre este terreno que la estimulación cardiaca se abre paso. Resulta arduo hacer recomendaciones en torno a la estimulación cardiaca en el manejo del síncope neuralmente mediado, dadas las limitaciones metodológicas de los estudios existentes, las cuales van desde la poca precisión de los resultados, hasta la terminación temprana, pasando por la ausencia de enmascaramientos. Después de mostrar un beneficio promisorio en registros, los estudios controlados iniciales fallaron en mostrar un beneficio real sobre la terapia convencional ${ }^{18-20}$.

La bradicardia o el componente cardioinhibitorio del síncope mediado neuralmente es el objetivo de la terapia de estimulación cardiaca. Dos tipos de síncope mediados neuralmente han sido el centro de la terapia con estimulación: el síncope vasovagal y el síndrome del seno carotídeo ${ }^{21}$.

Las guías son claras en establecer la indicación del implante de dispositivos en presencia de síncope y bloqueo AV de ॥ grado o bloqueo AV avanzado o completo y con menor grado de recomendación en casos de síncope en presencia de bloqueo de rama y estudio electrofisiológico positivo y en pacientes con síncope inexplicable con bloqueo de rama. En el síncope neuralmente mediado la recomendación es Ilb con nivel de evidencia $B$, y está prevista en los casos de síncope recurrente en mayores de 40 años con respuesta cardioinhibitoria en la prueba de mesa basculante después de que la terapia de primera línea ha fallado ${ }^{22}$.

A principios de los noventa se realizaron los primeros intentos de tratamiento del síncope neuralmente mediado con estimulación cardiaca en los pacientes con síntomas refractarios al manejo médico y a las recomendaciones no farmacológicas, lo anterior bajo la premisa de la existencia de un componente cardioinhibitorio en este grupo de pacientes.

Los estudios de estimulación pueden ser clasificados en 3 categorías: estimulación contra terapia estándar, estimulación contra placebo y estimulación convencional contra sistema CLS.

\section{Estudios de estimulación frente a terapia convencional}

En el primer estudio, publicado por Fitzpatrick en 1991, se logró demostrar que la estimulación cardiaca secuencial atenuaba los síntomas y retrasaba el síncope durante la mesa basculante ${ }^{23}$. El primer estudio de terapia con estimulación convencional contra placebo fue el Vasovagal Pacemaker Study (VPS), el cual comparó en 27 pacientes, con bradicardia relativa durante una prueba de mesa basculante, la estimulación bicameral con algoritmo de respuesta de caída de la frecuencia cardiaca contra la terapia estándar. A un año de seguimiento, el $22 \%$ de los pacientes con estimulación seguían presentando síncope contra el 70\% de aquellos en terapia estándar; se halló una reducción del riesgo relativo de síncope del $85,4 \%{ }^{18}$. Fue suspendido antes 
de completar el tiempo de seguimiento programado por el beneficio encontrado, lo cual constituye una limitación además de no estar cegado ${ }^{18}$. A este estudio le siguieron 2 trabajos con características similares: el Vasovagal Syncope International study (VASIS) y el Syncope Diagnosis and Treatment Study (SYDIT). El primero incluyó 42 pacientes con 3 o más episodios de síncope en los 2 últimos años, con respuesta cardioinhibitoria en la prueba de mesa basculante, y encontró una recaída en el $61 \%$ y en el $5 \%$ de los pacientes que no recibieron el dispositivo y en quienes sí lo hicieron, respectivamente. El segundo reportó una recaída del $4,3 \%$ en los que recibieron dispositivos, contra el $25,5 \%$ en el grupo de terapia médica; también fue suspendido de forma temprana debido a estos hallazgos. Estos primeros estudios motivaron extender el empleo de dispositivos de estimulación cardiaca al manejo del síncope neuralmente mediado con respuesta cardioinhibitoria ${ }^{19,24}$.

\section{Estimulación contra placebo}

Los hallazgos de los estudios anteriores llevaron a plantear estudios controlados con placebo y cegados. Son 3 los estudios con estas características: el Vasovagal Pacemaker Study II (VPS II), el Vasovagal Syncope and Pacing Trial (SYNPACE) y el Third International Study on Syncope of Unknown Etiology (ISSUE 3). Los 3 emplearon estimulación de 2 cámaras con algoritmo de caída de frecuencia cardiaca (DDD-RDR) frente a estimulación placebo (ODO) ${ }^{20,25,26}$.

El VPS II incluyó 100 pacientes con al menos 6 episodios de síncope o 3 o más en los 2 últimos años y una prueba de mesa basculante positiva pero sin definir una frecuencia cardiaca mínima durante la prueba. Se distribuyeron de manera aleatoria al modo de estimulación en DDD o al modo de monitor en ODO. A 6 meses de seguimiento no se lograron demostrar diferencias en las tasa de síncope ${ }^{20}$. La recurrencia de síncope se documentó en un $42 \%$ del grupo placebo y en un $33 \%$ del grupo con estimulación $(p=0,14)$. Entre tanto, en el SYNPACE se incluyeron 29 pacientes con el mismo número de episodios sincopales que en el VPS II, pero además de un síncope espontáneo tenían asistolia en la prueba de mesa basculante $(52 \%)$ o respuesta mixta $(48 \%)$. Los resultados mostraron recurrencia de síncope en el 38\% del grupo placebo y en el $50 \%$ del grupo estimulado.

Con el advenimiento del uso de monitores de eventos implantables se encontró una pobre correlación entre los resultados de la mesa basculante y el síncope espontáneo, mostrándose así que hasta el $50 \%$ de los pacientes con respuesta vasodilatadora en la mesa basculante presentaron asistolia severa durante el síncope, y que por tanto la mesa basculante no es el mejor criterio para la selección de los pacientes. La estrategia de manejo basada en los resultados del monitor de eventos se probó inicialmente en el estudio ISSUE-2, en el cual se implantaron marcapasos bicamerales a aquellos pacientes con pausas mayores de $3 \mathrm{~s}$ con síntomas y a las mayores de $6 \mathrm{~s}$ sin síntomas, para hallar una reducción del riesgo relativo del $57 \%$, y una reducción absoluta del riesgo de $32 \%(p<0,05)^{27}$.

El ISSUE 3, incluyó 511 pacientes mayores de 40 años, con al menos 3 episodios sincopales presuntamente mediados neuralmente en los últimos 2 años, a quienes se les implantó un monitor de eventos. De estos, 89 pacientes presentaron síncope con documentación de asistolia en el monitor de eventos y 77 fueron aleatorizados a estimulación contra placebo. El síncope recurrió en el $57 \%$ de los pacientes en el grupo placebo y en el $25 \%$ del grupo con estimulación $(p=0,039)^{26}$.

\section{Estimulación convencional frente a sistema de asa cerrada}

El método ideal para obtener un sistema de marcapasos fisiológico sería integrar el dispositivo al sistema cardiovascular, concepto que ha sido implementado en los sistemas CLS, los cuales convierten la información del sistema circulatorio aplicado al ventrículo derecho en concordancia con la frecuencia cardiaca.

El algoritmo CLS mide la impedancia ventricular latido a latido durante la fase sistólica del ciclo cardiaco y es capaz de detectar cambios tempranos en la contractilidad en la fase temprana del síncope vasovagal. Esta propiedad es la razón para la intervención temprana del CLS, de la cual se ha demostrado ocurre en un promedio de $4 \mathrm{~min}$ en la mesa basculante, mientras que el síncope aparece $14 \mathrm{~min}$ más tarde ${ }^{28}$.

La intervención en este momento suprime la bradicardia resultante y contrarresta la hipotensión producto de la vasodilatación, manteniéndose así el gasto cardiaco. No obstante, esta opción terapéutica se reserva para individuos altamente sintomáticos. Su ventaja frente a otros algoritmos de estimulación es la habilidad para compensar la respuesta de la frecuencia cardíaca en las fases iniciales del síncope vasovagal, cuando se detecta un incremento de la contractilidad pero cuando aún la cardioinhibición no se ha iniciado; el síncope vasovagal puede suprimirse debido al mantenimiento de un gasto cardiaco adecuado (la estimulación convencional únicamente se inicia cuando la cardioinhibición está presente y el gasto cardiaco ha caído $)^{29}$. Adicionalmente, en muchos pacientes la caída de la presión precede a la caída de la frecuencia cardiaca. En tal sentido, los marcapasos con sensores convencionales, que detectan únicamente los cambios en la frecuencia cardiaca, son capaces de manifestar la aparición de un episodio sincopal únicamente después de la caída de la frecuencia cardiaca, un evento que ocurre en la fase tardía de la fisiopatología del evento sincopal; por tanto, intervienen demasiado tarde, lo que limita su utilidad.

La mayoría de los estudios de estimulación en síncope vasovagal se basan en sistemas de estimulación fundados en el algoritmo de caída de frecuencia cardiaca, pero algunos muestran que el síncope vasovagal puede prevenirse de forma incompleta por la terapia con marcapasos estándar ${ }^{18,19}$, a pesar del desarrollo de sistemas de marcapasos más sofisticados con algoritmos para detectar las caídas de la frecuencia cardiaca, la cual marca el inicio del síncope cardioinhibitorio para prevenir el síncope vasovagal. Investigaciones previas en pacientes con síncope han demostrado que los cambios dinámicos en la contracción del miocardio ventricular usualmente preceden cualquier caída de la frecuencia cardiaca. El sistema CLS puede usar esta información para prevenir el síncope ${ }^{30}$.

El papel del sistema CLS en la prevención del síncope vasovagal ha sido establecido por Occhetta et al. en el 
estudio INotropy controlled pacing in VAsovagal SYncope (INVASY), y más recientemente por Kanjwal et al. Lo principal de ambos estudios fue el seguimiento relativamente corto, lo cual pone de manifiesto los posibles periodos asintomáticos largos, pudiendo reducir el impacto de los hallazgos $28,31,32$. El estudio más reciente con empleo de marcapasos con CLS para el manejo de síncope vasovagal con respuesta cardioinhibitoria en la mesa basculante se llevó a cabo en una población de pacientes sin otras comorbilidades, con un diseño cruzado, que involucró a 50 pacientes con un seguimiento de 36 meses y halló una reducción significativa en el número de síncopes y de presíncopes ${ }^{33}$.

De otra parte, el estudio más grande de marcapasos con CLS reunió 50 pacientes, con al menos 5 episodios sincopales en los últimos 2 años y prueba de mesa basculante positiva para síncope cardioinhibitorio. Aleatorizó a los pacientes a estimulación DDD-CLS contra DDI y encontró una recurrencia de síncope al año en el grupo de CLS del 0\%, frente al $78 \%$ en el grupo de DDI. La aleatorización fue suspendida a los 12 meses a la luz de estos resultados ${ }^{28}$.

Posteriormente comenzó la publicación de estudios con registros de pacientes comparando los 2 tipos de sensores. Uno de los primeros fue el de Kanjwal et al., donde en una muestra de 35 pacientes con síncope vasovagal recurrente y prueba de mesa basculante positiva para respuesta cardioinhibitoria se encontró una recurrencia de síncope del $59 \%$ contra el $83 \%$, cuando se compararon las 2 terapias a favor del sistema $\mathrm{CLS}^{32}$.

En fecha reciente se presentó el registro de Palmisano et al., que incluyó 41 pacientes con síncope vasovagal refractario y respuesta cardioinhibitoria en la prueba de mesa basculante, a quienes se les había implantado un marcapasos. De estos, 25 pacientes recibieron un marcapasos con sistema CLS y 16 un algoritmo convencional para prevención de síncope. Durante el seguimiento un paciente (1\%) del grupo con marcapasos con CLS y 6 (36\%) del grupo de marcapasos convencional $(p=0,01)$ desarrollaron síncope ${ }^{34}$.

Para la misma fecha se presentó el registro de Bortnik et al., en el cual se incluyeron 35 pacientes con síncope y presíncope antes de la implantación del CLS. El número de síncopes antes de la implantación del CLS fue en promedio de 6. En el seguimiento, 29 de 35 pacientes (83\%) eran asintomáticos; uno reportó persistencia del síncope pero mejoría en la calidad de vida. En todos los pacientes el número de síncopes posterior al implante del CLS fue significativamente menor. Los autores concluyeron que el sensor de CLS es una herramienta significativamente útil en el manejo del síncope vasovagal recurrente ${ }^{35}$.

Cada vez se cuenta con más evidencia que apoya el empleo de dispositivos de estimulación cardiaca en pacientes altamente seleccionados con síncope neuralmente mediado, en especial con sensores de tipo CLS. Aunque faltan estudios aleatorizados, con diseño multicéntrico y mayor número de pacientes, los marcapasos con CLS se perfilan como una alternativa de manejo en este tipo de pacientes, en quienes las opciones terapéuticas son escasas.

\section{Conflicto de intereses}

Los autores declaran no tener ningún conflicto de intereses.

\section{Bibliografía}

1. Rosanio S, Schwarz ER, Ware DL, Vitarelli A. Syncope in adults: Systematic review and proposal of a diagnostic and therapeutic algorithm. Int J Cardiol. 2013;162:149-57.

2. Soteriades ES, Evans JC, Larson MG, Chen MH, Chen L, Benjamin EJ, et al. Incidence and prognosis of syncope. N Engl J Med. 2002;347:878-85.

3. Sarasin FP, Louis-Simonet M, Carballo D, Slama S, Rajeswaran A, Metzger JT, et al. Prospective evaluation of patients with syncope: a population-based study. Am J Med. 2001;111: 177-84.

4. Sun BC, Emond JA, Camargo CA Jr. Direct medical costs of syncope-related hospitalizations in the United States. Am J Cardiol. 2005;95:668-71.

5. Edvardsson N, Frykman V, van Mechelen R, Mitro P, MohiiOskarsson A, Pasquié J-L, et al. Use of an implantable loop recorder to increase the diagnostic yield in unexplained syncope: results from the PICTURE registry. Europace. 2011;13: 262-9.

6. Brignole M, Vardas P, Hoffman E, Huikuri H, Moya A, Ricci $\mathrm{R}$, et al. Indications for the use of diagnostic implantable and external ECG loop recorders. Europace. 2009;11: 671-87.

7. Van Dijk N, Sprangers MA, Colman N, Boer KR, Wieling W, Linzer M. Clinical factors associated with quality of life in patients with transient loss of consciousness. J Cardiovasc Electrophysiol. 2006;17:998-1003.

8. Bartoletti A, Fabiani P, Bagnoli L, Cappelletti C, Cappellini M, Nappini G, et al. Physical injuries caused by a transient loss of consciousness: Main clinical characteristics of patients and diagnostic contribution of carotid sinus massage. Eur Heart J. 2008;29:618-24.

9. Davies AJ, Kenny RA. Falls presenting to the accident and emergency department: Types of presentation and risk factor profile. Age Ageing. 1996;25:362-6.

10. Linzer M, Yang EH, Estes NA, Wang P, Vorperian VR, Kapoor WN. Diagnosing syncope. Part 1: Value of history, physical examination, and electrocardiography. Clinical Efficacy Assessment Project of the American College of Physicians. Ann Intern Med. 1997;126:989-96.

11. Van Dijk N, Boer KR, Colman N, Bakker A, Stam J, van Grieken JJM, et al. High diagnostic yield and accuracy of history, physical examination, and ECG in patients with transient loss of consciousness in FAST: The Fainting Assessment study. J Cardiovasc Electrophysiol. 2008;19:48-55.

12. Epstein AE, DiMarco JP, Ellenbogen KA, Estes NAM, Freedman RA, Gettes LS, et al. ACC/AHA/HRS 2008 Guidelines for DeviceBased Therapy of Cardiac Rhythm Abnormalities: A report of the American College of Cardiology/American Heart Association Task Force on Practice Guidelines (Writing Committee to Revise the ACC/AHA/NASPE 2002 Guideline Update for Implantation of Cardiac Pacemakers and Antiarrhythmia Devices) developed in collaboration with the American Association for Thoracic Surgery and Society of Thoracic Surgeons. J Am Coll Cardiol. 2008;51:e1-62.

13. Sud S, Massel D, Klein GJ, Leong-Sit P, Yee R, Skanes AC, et al. The expectation effect and cardiac pacing for refractory vasovagal syncope. Am J Med. 2007;120:54-62.

14. Brignole M, Menozzi C, Bartoletti A, Giada F, Lagi A, Ungar A, et al. A new management of syncope: Prospective systematic guideline-based evaluation of patients referred urgently to general hospitals. Eur Heart J. 2006;27:76-82.

15. Jardine DL. Vasovagal syncope: New physiologic insights. Cardiol Clin. 2013;31:75-87.

16. Jhanjee R, Can I, Benditt DG. Syncope. Dis-Mon. 2009;55: 532-85. 
17. Medow MS, Stewart JM, Sanyal S, Mumtaz A, Sica D, Frishman WH. Pathophysiology, diagnosis, and treatment of orthostatic hypotension and vasovagal syncope. Cardiol Rev. 2008;16: 4-20.

18. Connolly SJ, Sheldon R, Roberts RS, Gent M. The North American Vasovagal Pacemaker Study (VPS). A randomized trial of permanent cardiac pacing for the prevention of vasovagal syncope. J Am Coll Cardiol. 1999;33:16-20.

19. Sutton R, Brignole M, Menozzi C, Raviele A, Alboni P, Giani $P$, et al. Dual-chamber pacing in the treatment of neurally mediated tilt-positive cardioinhibitory syncope: pacemaker versus no therapy: a multicenter randomized study. The Vasovagal Syncope International Study (VASIS) Investigators. Circulation. 2000;102:294-9.

20. Connolly SJ, Sheldon R, Thorpe KE, Roberts RS, Ellenbogen KA, Wilkoff BL, et al. Pacemaker therapy for prevention of syncope in patients with recurrent severe vasovagal syncope: Second Vasovagal Pacemaker Study (VPS II): A randomized trial. JAMA. 2003;289:2224-9.

21. Benditt DG, Adkisson WO. Approach to the patient with syncope: venues, presentations, diagnoses. Cardiol Clin. 2013;31:9-25.

22. Brignole M, Auricchio A, Baron-Esquivias G, Bordachar P, Boriani G, Breithardt O-A, et al. 2013 ESC Guidelines on cardiac pacing and cardiac resynchronization therapy: The Task Force on cardiac pacing and resynchronization therapy of the European Society of Cardiology (ESC). Developed in collaboration with the European Heart Rhythm Association (EHRA). Eur Heart J. 2013;34:2281-329.

23. Fitzpatrick A, Theodorakis G, Ahmed R, Williams T, Sutton R. Dual chamber pacing aborts vasovagal syncope induced by head-up 60 degrees tilt. Pacing Clin Electrophysiol PACE. 1991;14:13-9.

24. Ammirati F, Colivicchi F, Santini M, Syncope Diagnosis and Treatment Study Investigators. Permanent cardiac pacing versus medical treatment for the prevention of recurrent vasovagal syncope: A multicenter, randomized, controlled trial. Circulation. 2001;104:52-7.

25. Raviele A, Giada F, Menozzi C, Speca G, Orazi S, Gasparini G, et al. A randomized, double-blind, placebo-controlled study of permanent cardiac pacing for the treatment of recurrent tiltinduced vasovagal syncope. The vasovagal syncope and pacing trial (SYNPACE). Eur Heart J. 2004;25:1741-8.
26. Brignole M, Menozzi C, Moya A, Andresen D, Blanc JJ, Krahn AD, et al. Pacemaker therapy in patients with neurally mediated syncope and documented asystole: Third International Study on Syncope of Uncertain Etiology (ISSUE-3): A randomized trial. Circulation. 2012;125:2566-71.

27. Brignole M, Sutton R, Menozzi C, Garcia-Civera R, Moya A, Wieling W, et al. Early application of an implantable loop recorder allows effective specific therapy in patients with recurrent suspected neurally mediated syncope. Eur Heart J. 2006;27:1085-92.

28. Occhetta E, Bortnik M, Audoglio R, Vassanelli C, INVASY Study Investigators. Closed loop stimulation in prevention of vasovagal syncope. Inotropy Controlled Pacing in Vasovagal Syncope (INVASY): A multicentre randomized, single blind, controlled study. Europace. 2004;6:538-47.

29. Lau C-P, Tse H-F, Camm AJ, Barold SS. Evolution of pacing for bradycardias: sensors. Eur Heart J. 2007;9 Suppl I:I11-22.

30. Schaldach $\mathrm{M}$, Hutten $\mathrm{H}$. Intracardiac impedance to determine sympathetic activity in rate responsive pacing. Pacing Clin Electrophysiol PACE. 1992;15 11 Pt 2:1778-86.

31. Occhetta E, Bortnik M, Vassanelli C, INVASY Italian Feasibility Study Group. The DDDR closed loop stimulation for the prevention of vasovagal syncope: Results from the INVASY prospective feasibility registry. Europace. 2003;5:153-62.

32. Kanjwal K, Karabin B, Kanjwal Y, Grubb BP. Preliminary observations on the use of closed-loop cardiac pacing in patients with refractory neurocardiogenic syncope. J Interv Card Electrophysiol. 2010;27:69-73.

33. Russo V, Rago A, Papa AA, Golino P, Calabrò R, Russo MG, et al. The effect of dual-chamber closed-loop stimulation on syncope recurrence in healthy patients with tilt-induced vasovagal cardioinhibitory syncope: A prospective, randomised, single-blind, crossover study. Heart Br Card Soc. 2013;99:1609-13.

34. Palmisano P, Zaccaria M, Luzzi G, Nacci F, Anaclerio M, Favale S. Closed-loop cardiac pacing vs. conventional dual-chamber pacing with specialized sensing and pacing algorithms for syncope prevention in patients with refractory vasovagal syncope: Results of a long-term follow-up. Europace. 2012;14:1038-43.

35. Bortnik M, Occhetta E, dell'Era G, Secco GG, Degiovanni A, Plebani L, et al. Long-term follow-up of DDDR closed-loop cardiac pacing for the prevention of recurrent vasovagal syncope. J Cardiovasc Med (Hagerstown). 2012;13:242-5. 